\title{
LINEAR AND RICCATI MATRIX EQUATIONS
}

\section{LLOYD K. WILLIAMS}

Department of Mathematics

Texas Southern University

Houston, Texas 77004

(Received July 1, 1986 and in revised form April 14, 1987)

ABSTRACT. In this paper we find exact solutions for linear ordinary differential equations of any order when they are given in matrix form, as well as for classes of Riccati matrix equations with two or three arbitrary matrix coefficients. Other nonlinear systems of triangular form are also solved completely.

KEY WORDS AND PHRASES. Variation of constants technique, associated inatrix equations, linear and Riccati matrix equations. 1980 AMS SUBJECT CLASSIFICATION CODE. 34A05

1. INTRODUCTION.

A linear equation of finite order can be written as a system having a number of dependent variables equal to the order. Letting each successive one be the derivative of its predecessor, the number of equations will be the same as the order. This can then be put in matrix form.

For example, the linear equation of order $n$

$$
x^{(n)}=a_{0}(t) x+a_{1}(t) x^{\prime}+\ldots+a_{n-1}(t) x^{(n-1)}
$$

is equivalent to the system

$$
\left(\begin{array}{l}
x_{1}^{\prime}=x_{2} \\
x_{2}^{\prime}=x_{3} \\
x_{n}^{\prime}=a_{0} x_{1}+a_{1} x_{2}+\ldots+a_{n-1} x_{n}
\end{array}\right)
$$

which can be written as

$$
X^{\prime}=A X
$$

where $X=\left(x_{1}, x_{2}, \ldots, x_{n}\right)$ and $A=\left(\begin{array}{cccccc}0 & 1 & . & . & . & 0 \\ 0 & 0 & 1 & . & . & \\ 0 & 0 & . & . & . & 1 \\ a_{0} & a_{1} & \cdots & . & . & a_{n-1}\end{array}\right)$.

We will also treat Riccati matrix equations with two or three arbitrary coefficients and some other nonlinear systems. 
2. LINEAR ORDINARY DIFFERENTIAL EQUATIONS.

For convenience we will assume that all matrices are $n \times n$ with elements which are continuous functions of the same variable throughout this section.

The general solution of the linear equation

$$
X^{\prime}=A X+B
$$

with matric coefficients $A, B$ can be found from a nonsingular solution $Y$ of the corresponding homogeneous equation

$$
\mathrm{X}^{\prime}=\mathrm{AX}
$$

by the variation of constants technique as follows: Substitute $X=Y C$ in (2.1) to get

$$
Y^{\prime} C+Y C^{\prime}=A Y C+Y C^{\prime}=A Y C+B \text {. }
$$

From (2.3) we get

$$
\begin{gathered}
Y C^{\prime}=B \\
C=\int Y^{-1}(s) B(s) d s+c \\
X=Y\left(\int Y^{-1}(s) B(x) d x+c\right)
\end{gathered}
$$

Thus it suffices to consider equation of homogeneous forms.

- Now when $A=T$ is a triangular matrix we have the following.

THEOREM 2.1. The equation

$$
X^{\prime}=T X, \quad T=\left(t_{i j}(x)\right)
$$

is solvable by quadratures if $\mathrm{T}$ is a triangular matrix with all zeroes above the main diagonal.

PROOF. Consider the equation

$$
y^{\prime}=\mathrm{Ty}
$$

where $y$ is a vector. We solve the first equation $y_{1}^{\prime}=t_{11}(x) y_{1}$ for $y_{1}$. Using $y_{1}$ we then solve the next equation $y_{2}^{\prime}=t_{12}(x) y_{1}+t_{22}(x) y_{2}$, etc. In each instance we solve a simple linear first-order equation and in each case we find all solutions. Take $\mathrm{n}$ linearly independent solutions of (2.8) and write them as $n$ columns. This matrix, called a fundamental matrix of solutions, is a solution of the associated matrix equation (2.7). In the remainder of this section the independent variable $x$ will be understood but not written. Other results for triangular matrices include

THEOREM 2.2. If $S$ is invertible, $Q$ is nonsingular and $S^{-1} Q S$ is triangular, then the equation

$$
S X^{\prime}=Q S X+T
$$

is solvable by quadratures for any matrix $T$.

PROOF. Let $S^{-1} Q S=E$, a triangular matrix. Multiplying (2.9) by $S^{-1}$ we get

$$
X^{\prime}=E X+S^{-1} T \text {. }
$$

First we can solve

$$
X^{\prime}=E X
$$

by Theorem (2.1).

Then we solve (2.10) by variation of constants.

THEOREM 2.3. If $A$ is invertible, $B$ is nonsingular and $A^{-1} B$ is triangular, we can solve

$$
\mathrm{AX}^{\prime}=\mathrm{BX}+\mathrm{C}
$$

for any $C$. 
PROOF. Multiply (2.12) by $\mathrm{A}^{-1}$ and proceed as in proof of Theorem 2.2. COROLLARY 2.4. If $A$ is invertible $B$ is nonsingular and $A^{-1} B$ is trinagular, we can solve

$$
A^{\prime}=B X \text {. }
$$

We will say a matrix is continuous if each of its elements is a continuous function. In the next theorem we assume the matrix is continuous.

We now state and prove the main result of this paper.

THEOREM 2.5. Let $F$ be an invertible, continuous matrix. Then the equation

$$
Y^{\prime}=F Y
$$

is solvable by quadratures.

PROOF. We begin with the following.

LEMMA 2.6. Let $\mathrm{T}$ be a nonsingular triangular matrix and let $\mathrm{S}$ be a nonsingular matrix. The system

$$
\left(\begin{array}{l}
X^{\prime}=Y \\
Y=T X-S
\end{array}\right)
$$

is solvable by quadratures.

PROOF OF LEMMA 2.6. First we note that

$$
X^{\prime}=\mathrm{TX}-\mathrm{S}
$$

The solution of (2.16) can be obtained from the solutions of Equation (2.7) by Lagrange's variation-of-constants method referred to in the proof of Theorem (2.2).

Each solution of (2.16) yields a unique solution of the system (2.15), since $T$ is non-singular, by use of the relation

$$
\mathrm{Y}=\mathrm{TX}-\mathrm{S} \text {. }
$$

This completes the proof.

For statement of the next lemma let us assume momentarialy that TU-B is nonsingular for some matrices $U$ and $B$.

LEMMA 2.7. For any matrices $A, B, U, V$ and $W$ the pair of matrices

$$
X=U V+A W, \quad Y=U+B V+W
$$

is a solution of the system (2.15) if and only if:

$$
\begin{aligned}
& \left\{U(T U-B)^{-1} U+U(T U-B)^{-1} S+\left[A+U(T U-B)^{-1}(I-T A)\right] W\right\}^{\prime} \\
& =\left[I+B(T U-B)^{-1}\right] U+B(T U-B)^{-1} S+\left[I+B(T U-B)^{-1}(I-T A)\right] W .
\end{aligned}
$$

Here $I$ is the identity matrix.

PROOF OF LEMMA 2.7. (IF) Assuming that (2.18) holds we first define $V$ by the equation

$$
V=(T U-B)^{-1} U+(T U-B)^{-1} S+(T U-B)^{-1}(I-T A) W
$$

Multiply (2.19) by TU-B and simplify to get

$$
U+B V+W=T(I V+A W)-S
$$


Define $X, Y$ by

$$
\left(\begin{array}{l}
X=U V+A W \\
Y=U+B V+W
\end{array}\right)
$$

to get

$$
Y=T X-S
$$

Then note that $(2.18)$ can be written as

$$
X^{\prime}=Y .
$$

(ONLY IF). On the other hand, if (2.21) satisfies the system(2.15) we eliminate V from the equation

$$
U+B V+W=T(U V+A W)-S
$$

and substitute its value in (2.21).

We then see that the equation (2.18) is equivalent to (2.23). This completes the proof.

PROOF OF THEOREM 2.5. By the foregoing we may assume that $F$ is not triangular. As usual $|Z|$ will denote the determinant of $Z$ and $I$ stands for the identity.

Since $F$ is nonsingular there is a nonsingular $A_{1}$ such that $F A_{1}-I$ is nonsingular and a lower triangular $T_{1}=\left(t_{i j}\right)$ such that $T_{1} A_{1}-I$ is also nonsingular. This is accomplished by imposing nonvanishing restrictions on $\left|T_{1} A_{1}-I\right|$ and $\left|F A_{1}-I\right|$ as in the Example below.

Define $P$ by the equation

$$
\left(F A_{1}-I\right)\left(T_{1} A_{1}-I\right)^{-1}=P \text {. }
$$

Now since $F \neq T_{1}$ and $P$ is nonsingular, $F-P T_{1}$ is nonzero and in fact the coefficient of $t_{11}$ is nonzero in $\left|\mathrm{F}-\mathrm{PT}{ }_{1}\right|$. Accordingly, F-PT 1 can be made nonsingular by restricting $t_{11}$.

Let $C_{1}$ be such that $C_{1}-A_{1}, T_{1} C_{1}-I$ are nonsingular. The nonsingularity of $(I-P)-\left(F-P T T_{1}\right) C_{1}$ can be obtained from that of $F-P T_{1}$ by restricting an element of $C_{1}$.

Let $B_{1}$ be such that the pair $U=A_{1}+C_{1} B_{1}, B=B_{1}$ is a solution of

$$
(\mathrm{FU}-\mathrm{B})\left(\mathrm{T}_{1} \mathrm{U}-\mathrm{B}\right)^{-1}=\mathrm{P} \text {. }
$$

The value of $B_{1}$ must be $\left[(I-P)-\left(F-P T{ }_{1}\right) C_{1}\right]^{-1}\left(F-P T T_{1}\right) A_{1}$. Since $\left(\mathrm{T}_{1} \mathrm{C}_{1}-\mathrm{I}\right)\left[(\mathrm{I}-\mathrm{P})-\left(\mathrm{F}-\mathrm{PT}{ }_{1}\right) \mathrm{C}_{1}\right]^{-1}$ is nonsingular an element of $\mathrm{F}-\mathrm{PT} \mathrm{l}_{1}$ can be chosen so that

$$
\mathrm{T}_{1}+\left(\mathrm{T}_{1} \mathrm{C}_{1}-\mathrm{I}\right)\left[(\mathrm{I}-\mathrm{P})-\left(\mathrm{F}-\mathrm{PT} \mathrm{T}_{1}\right) \mathrm{C}_{1}\right]^{-1}\left(\mathrm{~F}-\mathrm{PT} \mathrm{T}_{1}\right)
$$

will be nonsingular. Then $T_{1} A_{1}+\left(T_{1} C_{1}-I\right) B_{1}$ is nonsingular and moreover $(2.26)$ is satisfied by $U=A_{1}+C_{1} B_{1}, B=B_{1}$. Finally an element of $F-P T$ can be chosen so that $I+\left(C_{1}-A\right)\left[(I-P)-\left(F-P T T_{1}\right) C_{1}\right]^{-1}(F-P T)$ is nonsingular. It follows that $A_{1}+\left(C_{1}-A_{1}\right) B_{1}$ is nonsingular.

At this point we assume that all choices have been made for the elements of $A_{1}$, $\mathrm{T}_{1}$ and $\mathrm{C}_{1}$.

In summary, the nonsingularity of $\mathrm{FA}_{1}-\mathrm{I}, \mathrm{T}_{1} \mathrm{~A}_{1}-\mathrm{I}$ is obtained by restricting a single element of $A_{1}$; nonsingularity of $F_{-P T}$ by restricting $t_{11}$; nonsingularity of $C_{1}-A_{1}, T_{1} C_{1}-I$ and $(I-P)-\left(F-P T T_{1}\right) C_{1}$ by restricting elements of $C_{1}$ and finally in the last two cases by use of elements of F-PT ${ }_{1}$. That this is possible can be seen by 
requiring that the product of all eight (8) determinants be nonzero.

We define $S_{1}$ by the equation

$$
\mathrm{PS}_{1}=(\mathrm{I}-\mathrm{P}) \mathrm{U}, \quad \mathrm{U}=\mathrm{A}_{1}+\mathrm{C}_{1} \mathrm{~B}_{1} .
$$

REMARK. Equations (2.25), (2.26) and (2.27) imply that the last expression in (2.18) of Lemma 2.7 is the result of multiplying the first one on the left by $F$.

Now let $\left(X_{1}, Y_{1}\right)$ be a solution of (2.15) with $S=S_{1}, T=T_{1}$.

We then seek a pair $\left(V_{1}, W_{1}\right)$ such that

$$
\left(\begin{array}{c}
x_{1}=U V_{1}+A_{1} W_{1} \\
Y_{1}=U+B_{1} V_{1}+W_{1} \\
U=A_{1}+C_{1} B_{1}
\end{array}\right)
$$

Since $A_{1}+\left(C_{1}-A_{1}\right) B_{1}$ is nonsingular, we can solve for $V_{1}, W_{1}$.

By Lemma 2.7 we have that (2.18) is satisfied. This in conjunction with the Remark above yields that $U\left(T_{1} U-B_{1}\right)^{-1} U+U\left(T_{1} U-B_{1}\right)^{-1} S_{1}+\left[A_{1}+\left(T_{1} U-B_{1}\right)\left(I-T_{1} A_{1}\right)\right] W_{1}$ is a solution of the equation of the theorem.

EXAMPLE. Let $n=2$ and $F=\left(\begin{array}{ll}f_{1}(x) & f_{2}(x) \\ f_{3}(x) & f_{4}(x)\end{array}\right), \quad T_{1}=\left(\begin{array}{ll}t_{1} & 0 \\ t_{2} & t_{3}\end{array}\right)$. Take $A_{1}=\left(\begin{array}{ll}a_{1} & a_{2} \\ a_{3} & a_{4}\end{array}\right)$. Both quantities $\left|\mathrm{FA}_{1}-\mathrm{I}\right|$ and $\left|\mathrm{T}_{1} \mathrm{~A}_{1}-\mathrm{I}\right|$ can be made nonzero by restrictions on $\mathrm{a}_{1}$ as is readily seen by expanding them. Likewise setting $P=\left(\begin{array}{ll}p_{1} & p_{2} \\ p_{3} & p_{4}\end{array}\right)$ and expanding $|F-P T|$ the coefficient of $t_{1}$ is $|P| t_{3}-p_{1} f_{4}+p_{3} f_{2}$. In this connection we note in general that $\left|\mathrm{F}-\mathrm{PT}_{1}\right|=|\mathrm{P}|\left|\mathrm{P}^{-1} \mathrm{~F}-\mathrm{T}_{1}\right|$ since $\mathrm{P}$ is nonsingular.

We conclude this section with a result needed in the next.

THEOREM 2.9. Let $U$ be a nonsingular solution of $X^{\prime}=A X$ and let $V=U^{-1}$, then $V$ is a solution of $X^{\prime}=-X A$.

$$
\begin{array}{ll}
\text { PROOF. } & V U=I \\
V^{\prime} U & +V^{\prime} U=0 \\
V^{\prime} U & +V A U=0 \\
V^{\prime}=-V A .
\end{array}
$$

\section{RICCATI MATRIX EQUATIONS.}

Throughout this section all matrices are $n \times n$ matrices whose elements are functions of the same variable in each theorem. The theorems of the last section yield the following.

THEOREM 3.1. For any nonsingular matrices $F, G$ the equation

is solvable by quadratures.

$$
X^{\prime}+X F X+X G=0
$$

PROOF. For given $F, G$, let $A, B, C$ be nonsingular matrices such that

$$
F=C^{-1} B, \quad G=C^{-1} A C-C^{-1} C^{\prime}
$$

and consider the set of equations 


$$
\left(\begin{array}{l}
U^{\prime}=A U+B \\
U V=C
\end{array}\right)
$$

From (3.3) we get

$$
V^{\prime}+V C^{-1} B V+V\left(C^{-1} A C-C^{-1} C^{\prime}\right)=0
$$

so that

$$
V^{\prime}+V F V+V G=0 \text {. }
$$

To solve the given equation choose any $C$ and solve (3.2) for $A$ and $B$.

Use Theorem 2.5 of the last section to solve for $U$. from which we get $V$ from (3.3) .

THEOREM 3.2. For nonsingular $P, Q, R$ the equation

$$
\mathrm{X}^{\prime}+\mathrm{PX}+\mathrm{XQX}+\mathrm{XR}=0
$$

is solvable by quadratures.

PROOF. For given $P, Q, R$ let $F, G, H$ be such that

$$
H^{-1} H^{\prime}=P, \quad F H=Q, \quad G=R \text {. }
$$

Note that if $H W$ is a solution of

$$
\mathrm{X}^{\prime}+\mathrm{XFX}+\mathrm{XG}=0
$$

then $W^{\prime}+H^{-1} H^{\prime} W+W F H W+W G=0$. To solve the given equation, use Theorem 2.6 to find $H$ from the first equation of (3.4). Then get $F$ and $G$ from (3.4).

Finally, use the preceding theorem and its proof to get $\mathrm{HW}$, from which we determine $W$.

4. NONLINEAR SYSTEMS.

In the last section some nonlinear equations were solved. Let $X$ be a vector and let $F$ be a vector function. Call the system of differential equations

$$
X^{\prime}=F(X)
$$

triangular if the $k$-th component of $F$ is a function of the first $k$ components of $X$. THEOREM 4.1. If $X^{\prime}=F(X)$ is triangular we can solve it by quadratures.

PROOF. $\quad x_{1}^{\prime}=F_{1}\left(x_{1}\right)$ is solved by separation of variables. Then $x_{2}^{\prime}=F_{2}\left(x_{1}, x_{2}\right)$ is solved by the methods used in [1], $x_{3}$ and all other $x_{i}, i=4,5, \ldots, n$, are determined likewise.

\section{REFERENCES}

1. Williams, L. K. Nonlinear Differential Equations and Algebraic Systems, International Journal of Mathematics and Mathematical Sciences 1 , (1978), $257-267$.

2. Roxin, E. 0. Ordinary Differential Equations, Wadsworth, 1972.

3. Reid, W. T. Riccati Differential Equations, Academic Press, 1972. 


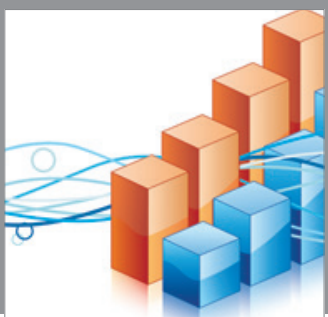

Advances in

Operations Research

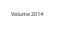

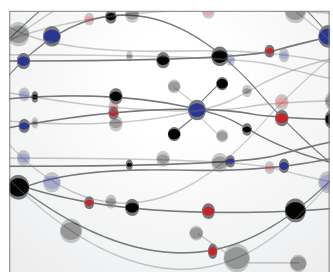

\section{The Scientific} World Journal
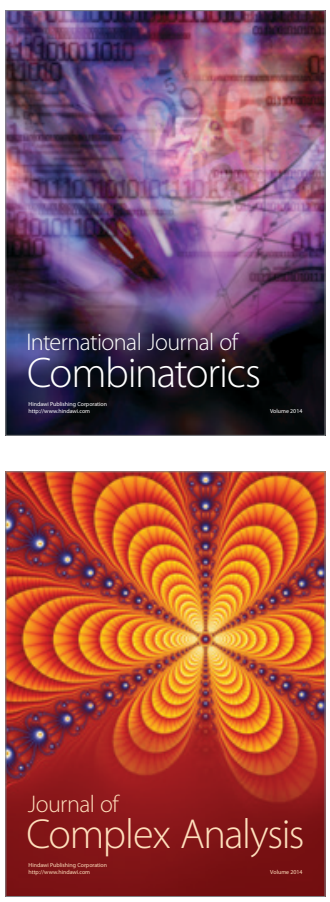

International Journal of

Mathematics and

Mathematical

Sciences
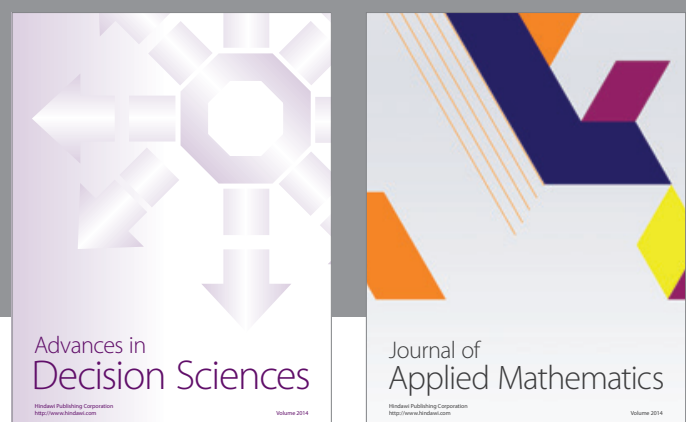

Journal of

Applied Mathematics
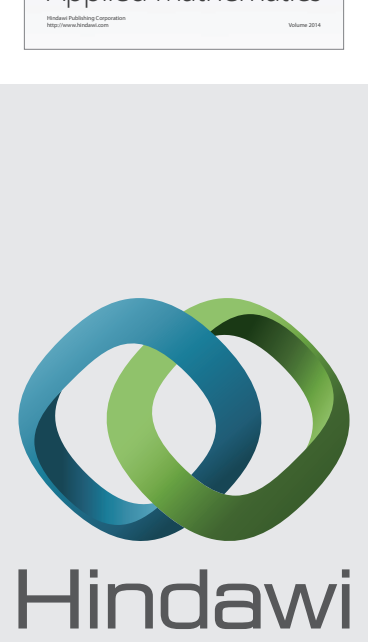

Submit your manuscripts at http://www.hindawi.com
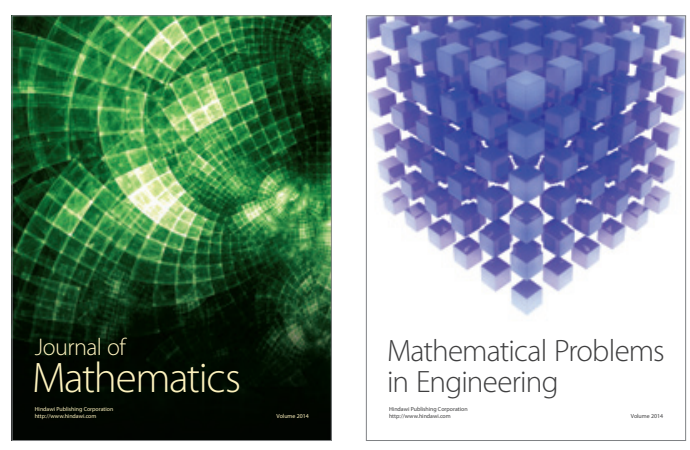

Mathematical Problems in Engineering
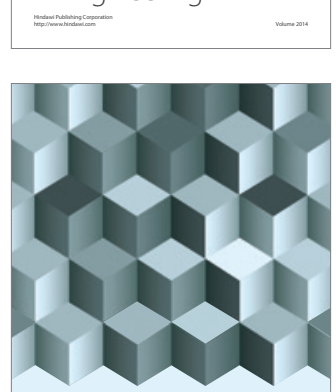

Journal of

Function Spaces
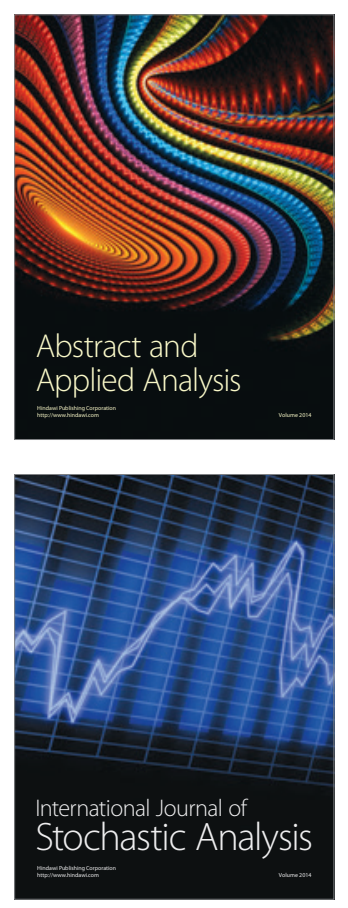

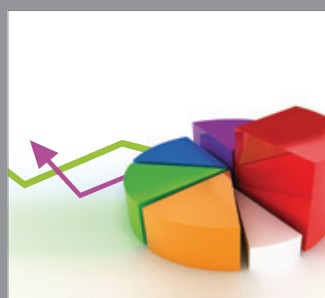

ournal of

Probability and Statistics

Promensencen
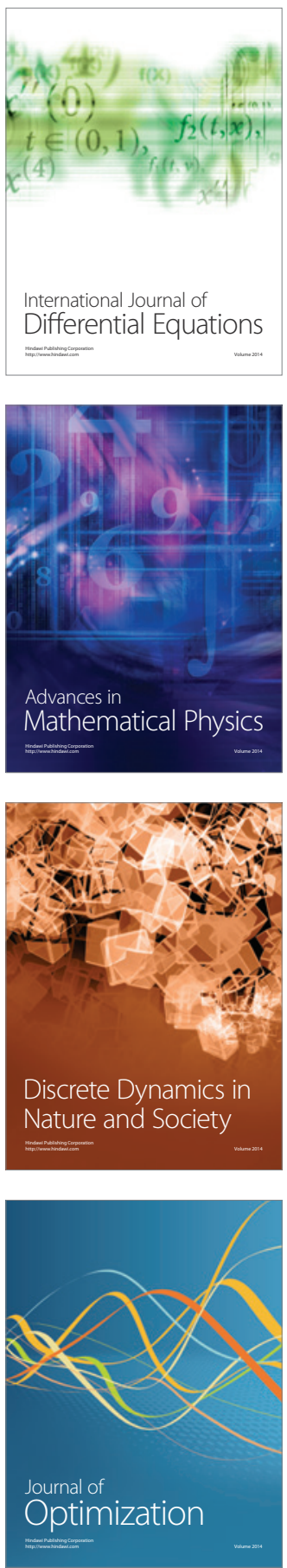